\title{
Rentabilidade de diferentes híbridos de milho, no Estado do Tocantins, safra 2009/2010
}

\begin{abstract}
RESUMO
Objetivou-se com o trabalho estimar os resultados econômicos de diferentes híbridos de milho, no Estado do Tocantins. Os experimentos foram realizados em seis ambientes, os quais foram constituídos pela combinação entre município, data de semeadura e dose de adubação nitrogenada em cobertura. $\mathrm{O}$ delineamento experimental em cada ambiente foi o de blocos ao acaso, posteriormente, seus componentes foram analisados de forma conjunta (diferenciando os ambientes), mediante análise de variância. Para a análise econômica foi considerado para efeito de cálculo, os custos com insumos, maquinários, mão-deobra, e outros itens envolvidos direta/indiretamente no processo produtivo, variando apenas o custo relativo a sementes e adubação nitrogenada. As produções de grãos obtidas em cada parcela determinaram à obtenção do resultado líquido. Na média, excetuando-se o híbrido simples modificado, o melhor híbrido de cada tipo foi classificado no grupo estatístico superior, sem diferença estatística entre eles, com médias de $2160 \mathrm{~kg} \mathrm{ha}^{-1}$ (melhor híbrido simples), $1915 \mathrm{~kg} \mathrm{ha}^{-1}$ (melhor híbrido duplo) e $2370 \mathrm{~kg}$ ha $^{-1}$ (melhor híbrido triplo). Os melhores híbridos simples, triplos e duplos apresentaram em média, adequada rentabilidade nas condições experimentais da presente pesquisa.
\end{abstract}

Palavras-chave: Zea mays, resultado líquido, adubação de cobertura.

\section{Lucrativeness of corn hybrids, in the State of Tocantins, crops 2009/2010}

\begin{abstract}
This research has aimed to esteem the economic results of different corn hybrids, in the Tocantins State. The experiments were conducted in six environments, which were formed by the combination of among city, date of sowing and nitrogen fertilizer on top dressing. The experimental design, in each environment, was the randomized blocks, and later, its components were submitted the thorough analysis (differentiating environments). At the economic analysis was considered for the purpose of calculation the cost of the inputs, machinery, labor, and other items directly/indirectly that were involved in the production process, and it's varying only the cost on the seed and nitrogen fertilization. Grain yields at each plot were used to determinate the net income. In average, the best hybrid of each one was classified in the superior statistic group, without statistic difference among them, with $2160 \mathrm{~kg} \mathrm{ha-1}$ (the best simple hybrid), $1915 \mathrm{~kg} \mathrm{ha}^{-1}$ (the best duple hybrid) and $2370 \mathrm{~kg} \mathrm{ha}^{-1}$ (the best triple hybrid). The best simple, double and triple hybrids had shown, in average, good lucrativeness at the experimental condition of this research.
\end{abstract}

Key-words: Zea mays, net income, fertilization coverage.

*Autora para correspondência

${ }^{1}$ Universidade Federal do Tocantins - UFT, Campus Universitário de Gurupi, *email: elianerotili@yahoo.com.br 


\section{INTRODUÇÃO}

A análise econômica tem como objetivo auxiliar os agricultores na tomada de decisão, sobretudo no que se refere ao que cultivar e como cultivar (Zanatta et al. 1993). Ao comparar a receita média ou o preço com os custos totais médios, obtém-se a análise econômica da atividade por unidade produtiva, identificando a lucratividade do empreendimento. No caso da análise operacional, ao comparar-se a receita média ou o preço com os custos operacionais, tem-se o conceito de resíduo ou margem (líquida ou bruta) de cada unidade produzida (Castro et al. 2006; Reis, 2002). Para Reis (2002) só haverá lucro econômico na atividade produtiva se o preço do produto ou receita média for maior que o custo unitário de produção. Neste caso, o bem produzido proporciona um retorno que supere os custos alternativos, que é a remuneração ao capital e ao trabalho empregados na atividade.

$\mathrm{O}$ nitrogênio é o nutriente exigido em maior quantidade pela cultura do milho, influenciando a resposta em produtividade de grãos, mas também onerando o custo de produção, o que, muitas vezes, determina a tomada de decisão quanto à dose, fonte e forma de aplicação (Cruz et al. 2008; Melo et al. 2011; Duete et al. 2009). Os fertilizantes nitrogenados podem representar $75 \%$ dos custos da adubação do milho, o que corresponde a cerca de $40 \%$ dos custos de produção da cultura (Lima, 2010). Para Carvalho et al. (2012) deve-se levar em consideração o uso racional dos recursos em termos de sustentabilidade ambiental, evitando desperdícios e contaminação do meio ambiente.

$\mathrm{O}$ incremento na produtividade de grãos envolve diversos fatores, destacando-se entre eles, a escolha correta de uma cultivar para o manejo em uma determinada região (Oliveira et al. 2012). No Brasil, a utilização de híbridos está associada ao tamanho da propriedade, à renda per capita do produtor, à infraestrutura e ao investimento em tecnologia; a escolha de híbridos pode estar associada ao preço das sementes (Rosinha, 2000).
A produção de sementes híbridas de milho é um dos avanços tecnológicos desenvolvidos para essa cultura, e a escolha adequada do híbrido torna-se fundamental para a obtenção de altas produtividades e em consequência elevada lucratividade das lavouras (Santos et al. 2002). Sangoi et al. (2004) encontraram influência da cultivar em relação à rentabilidade dos sistemas de produção, quanto ao nível de manejo. Os híbridos simples são potencialmente mais produtivos que os demais, apresentando maior uniformidade de plantas e espigas, porém são também os mais onerosos. Os híbridos triplos são uniformes e seu potencial produtivo é intermediário entre os híbridos simples e duplos. O mesmo ocorre com o preço de suas sementes. Os híbridos duplos são mais variáveis em características da planta e espiga que os simples e triplos. O custo da semente dos duplos é mais baixo que o preço da semente dos simples e triplos (Junqueira e Morabito, 2006).

É de grande importância o conhecimento dos diferentes tipos de genótipos, quanto ao melhoramento/biotecnologia envolvida, que utilizem eficientemente os nutrientes, uma vez que os fertilizantes estão entre os itens mais onerosos no sistema de produção de milho (Ceccarelli, 1996). Os diversos híbridos e variedades requerem quantidades diferentes de nitrogênio, de acordo com seu potencial de produtividade (Fernandes e Buzetti, 2005). Dessa forma, este trabalho teve como objetivo estimar os resultados econômicos de diferentes tipos de híbridos de milho, no Estado do Tocantins.

\section{MATERIAL E MÉTODOS}

Os experimentos foram realizados em seis ambientes no Estado do Tocantins, os quais foram constituídos pela combinação entre município, data de semeadura e dose de adubação nitrogenada em cobertura, conforme Tabela 1, avaliando-se híbridos comerciais de milho agrupados em Híbrido Simples (HS), Híbrido Simples Modificado (HSM), Híbrido Triplo (HT) e Híbrido Duplo (HD). 
Tabela 1. Especificação dos seis ambientes: município, localização, data de semeadura e dose de nitrogênio em cobertura, no Estado do Tocantins, safra 2009/2010.

\begin{tabular}{lcccc}
\hline Ambiente & Município & Localização & Nitrogênio em cobertura & Data de semeadura \\
\hline AMB1 & Gurupi I & $11^{\circ} 43^{\prime} \mathrm{S} ; 49^{\circ} 04^{\prime} \mathrm{W}$, & & $21 / \mathrm{nov} / 09$ \\
AMB2 & Gurupi II & $280 \mathrm{~m}$ & $0 \mathrm{~kg} \mathrm{ha}^{-1}$ & $14 / \mathrm{dez} / 09$ \\
AMB3 & Palmas & $1^{\circ} 23^{\prime} \mathrm{S} ; 48^{\circ} 22^{\prime} \mathrm{W} ;$ & & $03 / \mathrm{dez} / 09$ \\
\hline AMB4 & Gurupi I & $220 \mathrm{~m}$ & $21 / \mathrm{nov} / 09$ \\
AMB5 & Gurupi II & $11^{\circ} 43^{\prime} \mathrm{S} ; 49^{\circ} 04^{\prime} \mathrm{W}$, & & $14 / \mathrm{dez} / 09$ \\
AMB6 & Palmas & $280 \mathrm{~m}$ & $03 / \mathrm{dez} / 09$ \\
\hline
\end{tabular}

A adubação de semeadura utilizada foi a mesma, em todos os ambientes, com a aplicação de 600 $\mathrm{kg} \mathrm{ha}^{-1}$ da formulação NPK 04-14-08 + 0,3\% Zn. A adubação de cobertura, quando realizada, foi feita na dose de $144 \mathrm{~kg} \mathrm{ha}^{-1}$ de $\mathrm{N}$ entre os estágios V4 e V6. Os demais tratos culturais (controle de plantas daninhas e pragas - todos químicos) foram efetuados assim que se fizeram necessários $\mathrm{O}$ delineamento experimental em cada ambiente foi o de blocos ao acaso, com duas repetições, e em cada parcela experimental avaliaram-se as duas fileiras centrais de cinco metros de comprimento. Realizou-se a análise de variância, e foram consideradas as despesas com insumos, maquinários, mão-de-obra, e outros itens envolvidos direta/indiretamente no processo produtivo, como depreciação, despesas administrativas e juros, com base no relatório de informação trimestral da Superintendência Regional no Tocantins, do Banco da Amazônia S. A. (BASA, 2010), extraindo-se apenas o custo relativo a sementes e adubação nitrogenada, cabendo-se nestes componentes, os valores dos híbridos utilizados combinados com diferentes ambientes (adubação nitrogenada em cobertura), com valores médios no Tocantins.

As produções obtidas determinaram a obtenção da receita bruta, utilizando-se o preço do milho grão pago por saca ao produtor no momento da venda, baseado em dados da CONAB (2010), a partir dos preços, safra verão safras 2009/2010, no valor de $\mathrm{R} \$ 17,46$ a saca de $60 \mathrm{~kg}$. $\mathrm{O}$ resultado líquido foi determinado em cada parcela em $\mathrm{kg} \mathrm{ha}^{-1}$ pela receita bruta, extraindose o custo total, considerando as variações de híbridos (preço da semente no comércio local no momento da semeadura) e o custo do nitrogênio em cobertura. Os dados do resultado econômico foram então transformados para a realização da análise de variância conjunta dos experimentos e do teste de agrupamento de médias Scott-Knott a $5 \%$ de probabilidade, apresentando-se apenas o melhor e pior genótipo dentro de cada tipo de híbrido.

\section{RESULTADOS E DISCUSSÃO}

As médias dos resultados líquidos foram influenciadas significativamente pelos híbridos de milho em cada ambiente e, na análise conjunta, pelos híbridos, ambientes e interação entre ambos (Tabela 2). As diferenças nas rendas líquidas podem ser explicadas por diversos fatores, como por exemplo, custo de produção pelos diferentes valores de preços das sementes e produtividade de grãos dos híbridos nos diferentes ambientes. 
Tabela 2. Resultado líquido $\left(\mathrm{kg} \mathrm{ha}^{-1}\right)$ para híbridos de milho: simples (HS), simples modificado (HSM), duplos (HD) e triplos (HT) submetidos a seis ambientes (AMB), no Estado do Tocantins, safra 2009/2010

\begin{tabular}{|c|c|c|c|c|c|c|c|c|}
\hline \multirow{3}{*}{$\begin{array}{l}\text { Tipo } \\
\text { de } \\
\text { híbrido }\end{array}$} & \multirow{3}{*}{ Desempenho } & \multicolumn{7}{|c|}{ Resultado líquido $\left(\mathrm{kg} \mathrm{ha}^{-1}\right)$} \\
\hline & & \multicolumn{3}{|c|}{$0 \mathrm{~kg} \mathrm{ha}^{-1}$} & \multicolumn{4}{|c|}{$144 \mathrm{~kg} \mathrm{ha}^{-1}$} \\
\hline & & AMB 1 & AMB 2 & AMB 3 & AMB 4 & AMB 5 & AMB 6 & Média \\
\hline \multirow[b]{2}{*}{ HS } & Melhor & 3150 Аa & $1890 \mathrm{Ab}$ & $1500 \mathrm{Bb}$ & $1830 \mathrm{Ab}$ & $1890 \mathrm{Ab}$ & $2700 \mathrm{Ba}$ & $2160 \mathrm{~A}$ \\
\hline & Pior & $1140 \mathrm{Ca}$ & $1080 \mathrm{Ba}$ & $-1080 \mathrm{Cb}$ & $60 \mathrm{Bb}$ & $2100 \mathrm{Aa}$ & $0 \mathrm{Db}$ & $550 \mathrm{C}$ \\
\hline \multirow{2}{*}{ HSM } & Melhor & $1470 \mathrm{Cb}$ & $1110 \mathrm{Bb}$ & $600 \mathrm{Cb}$ & $2490 \mathrm{Aa}$ & $900 \mathrm{Bb}$ & $1800 \mathrm{Ca}$ & $1395 \mathrm{~B}$ \\
\hline & Pior & $810 \mathrm{Cb}$ & $1080 \mathrm{Bb}$ & $390 \mathrm{Cb}$ & $2010 \mathrm{Aa}$ & $1620 \mathrm{Ba}$ & $2160 \mathrm{Ba}$ & $1345 \mathrm{~B}$ \\
\hline \multirow{2}{*}{ HD } & Melhor & $1380 \mathrm{Cb}$ & $1380 \mathrm{Bb}$ & $1170 \mathrm{Bb}$ & $1320 \mathrm{Bb}$ & $2760 \mathrm{Aa}$ & $3480 \mathrm{Aa}$ & $1915 \mathrm{~A}$ \\
\hline & Pior & $2040 \mathrm{Ba}$ & $840 \mathrm{Bb}$ & 2040 Aa & $930 \mathrm{Bb}$ & $1050 \mathrm{Bb}$ & $210 \mathrm{Db}$ & $1185 \mathrm{~B}$ \\
\hline \multirow{2}{*}{ HT } & Melhor & $4020 \mathrm{Aa}$ & $1530 \mathrm{Bc}$ & $1320 \mathrm{Bc}$ & $2880 \mathrm{Ab}$ & $1890 \mathrm{Ac}$ & $2580 \mathrm{Bb}$ & $2370 \mathrm{~A}$ \\
\hline & Pior & $2190 \mathrm{Ba}$ & $900 \mathrm{Bb}$ & $120 \mathrm{Cb}$ & $3120 \mathrm{Aa}$ & $1050 \mathrm{Bb}$ & $540 \mathrm{Db}$ & $1320 \mathrm{~B}$ \\
\hline \multicolumn{2}{|c|}{ F - Híbridos (H) } & ** & * & *** & *** & ** & ${ }^{* * *}$ & \\
\hline \multicolumn{2}{|c|}{ F - Ambientes (A) } & - & - & - & - & - & - & \\
\hline \multicolumn{2}{|c|}{ F - Interação H x A } & - & - & - & - & - & - & \\
\hline & $\begin{array}{l}\text { Média } \\
\text { CV }(\%)\end{array}$ & 1656 a & $1590 \mathrm{a}$ & $918 \mathrm{~b}$ & $\begin{array}{r}1854 \text { a } \\
18,66 \\
\end{array}$ & 1848 a & 1698 a & $1596-$ \\
\hline
\end{tabular}

*, ** Diferenças significativas a 5\% e 1\% de probabilidade pelo teste F. Médias seguidas de mesmas letras maiúsculas na coluna e minúsculas na linha não diferem estatisticamente pelo teste Scott-Knott a 5\% de probabilidade, para produtividade.

Silva et al. (2004) afirmam que a diferença na produtividade de grãos entre cultivares de diferentes bases genéticas é acentuada à medida que se melhora o manejo. A utilização de híbridos simples nos sistemas de produção com alto investimento em manejo foi preconizada por Sangoi et al. (2004) como economicamente vantajosa, quando comparada com a utilização de híbridos duplos e variedades de polinização aberta.

$\mathrm{Na}$ maioria dos ambientes o melhor híbrido simples não diferiu estatisticamente do melhor híbrido triplo, o que também foi observado entre o melhor híbrido simples e o melhor híbrido duplo. Na média, excetuando-se o híbrido simples modificado, o melhor híbrido de cada tipo foi classificado no grupo estatístico superior, sem diferença estatística entre eles, com médias de $2160 \mathrm{~kg} \mathrm{ha}^{-1}$ (melhor híbrido simples), $1915 \mathrm{~kg} \mathrm{ha}^{-1}$ (melhor híbrido duplo) e $2370 \mathrm{~kg} \mathrm{ha}^{-1}$ (melhor híbrido triplo) (Tabela 2). Comparando os desempenhos de rendimentos de grãos de híbridos de milho, Emygdio et al. (2007) encontraram resultados semelhantes entre os melhores híbridos simples e triplos. Assim, a partir dos resultados desse experimento, alguns híbridos duplos ou triplos passam a ser uma boa opção para produtores que buscam maiores resultados líquidos, na média dos ambientes utilizados, já que neste trabalho os híbridos duplos e triplos possibilitaram retornos semelhantes a híbridos simples.

Segundo Emygdio et al. (2007), o custo médio de um híbrido duplo é $46 \%$ inferior ao de um híbrido simples, considerando os preços médios. Sangoi et al. (2006), na região de Santa Catarina avaliando o desempenho agronômico e econômico de cultivares de milho com níveis de variabilidade genética em sistemas de produção contrastantes quanto ao investimento em manejo, afirmou que a utilização de híbridos duplos é uma estratégia que pode ser economicamente interessante para produtores com tetos de rendimentos intermediários.

Resultados que podem reforçar esta idéia é de que o melhor híbrido duplo (1915 $\left.\mathrm{kg} \mathrm{ha}^{-1}\right)$ foi estatisticamente superior ao pior híbrido simples $\left(550 \mathrm{~kg} \mathrm{ha}^{-1}\right)$ na média dos ambientes, o que também aconteceu em cada ambiente.

Além disso, quando se compara os piores desempenhos, observa-se que na média dos ambientes o pior híbrido duplo (1185 kg ha $\left.{ }^{-1}\right)$ e triplo (1320 $\mathrm{kg} \mathrm{ha}^{-1}$ ) proporcionou resultados líquidos estatisticamente maiores que o pior híbrido simples $\left(550 \mathrm{~kg} \mathrm{ha}^{-1}\right)$. Isto concorda com a observação de Emygdio et al. (2007), que relatam a existência de híbridos simples com 
potencial aquém do esperado pelo tipo de híbrido.

Entre os ambientes, no ambiente 3 os híbridos em geral apresentaram estatisticamente menor média de resultado líquido, $918 \mathrm{~kg} \mathrm{ha}^{-1}$. Nos demais, não houve diferença estatística, porém, nos Ambientes 4 e 5 (Gurupi I e Gurupi II com nitrogênio em cobertura) os híbridos apresentaram os maiores valores de resultados líquidos, 1854 e $1848 \mathrm{~kg} \mathrm{ha}^{-1}$, respectivamente.

\section{CONCLUSÕES}

$\mathrm{O}$ risco de se obter resultados líquidos baixos, em ambientes providos de doses de nitrogênio maiores, nesta pesquisa, foi menor em relação aos ambientes com baixa dose de adubação nitrogenada.

Os melhores híbridos simples, triplos e duplos apresentaram em média, similares resultados líquidos nas condições experimentais da presente pesquisa.

\section{AGRADECIMENTOS}

Ao Conselho Nacional de Desenvolvimento Científico e Tecnológico - CNPq - Brasil, pelo apoio financeiro ao projeto e bolsa PIBIC.

A Coordenação de Aperfeiçoamento de Pessoal de Nível Superior - CAPES - Brasil, pela concessão de bolsas de mestrado e PNPD.

\section{REFERÊNCIAS}

BASA - BANCO DA AMAZÔNIA S. A. Relatório de informação trimestral sobre atividade agropecuária. Superintendência Regional no Tocantins, $2^{\circ}$ trimestre/2010.

Carvalho, E. V. de; Afférri, F. S; Dotto, M. A; Peluzio, J. M. Rotili, E. A; Almeida, P. B. de. Eficiência agronômica do uso do nitrogênio em genótipos de milho e correlação com caracteres agronômicos. Journal of Biotechnology and Biodiversity, v. 3, n.1, p. 68-73, 2012.

Castro, S. H; Reis, R. P; Lima, A. L. R. Custos de produção da soja cultivada sob sistema de plantio direto: estudo de multicasos no oeste da Bahia. Ciência e Agrotecnologia, v. 30, n. 6, p. 1146-1153, 2006.

Ceccarelli, S. Adaptation to low/high input cultivation. Euphytica, v. 92, n.1/2, p. 203-214, 1996. $\begin{array}{lcr}\text { CONAB - COMPANHIA NACIONAL } & \text { DE } \\ \text { ABASTECIMENTO. } & \text { Indicadores } & \text { da } \\ \text { Agropecuária. } & \text { Disponível } & \text { em: }\end{array}$ $<$ http://www.conab.gov.br/OlalaCMS/uploads/arquiv os/10_11_29_15_09_15_0508

_mandioca_e_milho.pdf>. Acesso em: 03 set. 2010.

Cruz, S. C. S; Pereira, F. R. da S; Santos, J. R; Albuquerque, A. W. de; Pereira, R. G. Adubação nitrogenada para o milho cultivado em sistema plantio direto, no Estado de Alagoas. Revista Brasileira de Engenharia Agrícola e Ambiental, v. 12, n. 1 p. 62-68, 2008.

Duete, R. R. C; Muraoka, T; Silva, E. C; Trevelin, P. C. O; Ambrosano, E. J. Viabilidade econômica de doses e parcelamentos da adubação nitrogenada na cultura do milho em Latossolo Vermelho Eutrófico. Acta Scientiarum Agronomy, v. 31, n. 1, p. 175181, 2009.

Emygdio, B. M; Ignaczak, J. C; Cargnelutti Filho, A. Potencial de rendimento de grãos de híbridos comerciais simples, triplos e duplos de milho. Revista Brasileira de Milho e Sorgo, v. 06, n. 1, p. 95-103, 2007.

Fernandes, F. C. S; Buzetti, S. Efeito de níveis de nitrogênio na produtividade de seis cultivares de milho (Zea mays L.). Revista Científica Eletrônica de Agronomia, v. 07, n. 01, 2005.

Junqueira, R. Á. R; Morabito, R. Um modelo de otimização linear para o planejamento agregado da produção e logística de sementes de milho. Revista Produção online. v. 16, n. 3, p. 510-525. 2006.

Lima, F. F. da. Bacillus subtilis e níveis de nitrogênio sobre o desenvolvimento e a produtividade do milho. Ano de obtenção: 2010. 52 p. Dissertação (Mestrado em Agronomia) Universidade Federal do Piauí, Teresina.

Melo, F. B; Corá, J. E; Cardoso, M. J. Fertilização nitrogenada, densidade de plantas e rendimento de milho cultivado no sistema plantio direto. Revista Ciência Agronômica, v. 42, n. 1, p. 27-31, 2011.

Oliveira, G. H. F; Arnhold, E; Araújo B. L; Silva, R. N. O; Costa, J. R. S; Oliveira Junior, E. A, et al. Produtividade de cultivares de milho em região préamazônica. Revista Trópica: Ciências Agrárias e Biológicas, v. 6, n. 2, p. 3-7, 2012.

Reis, R. P. Fundamentos de economia aplicada. Lavras: UFLA/FAEPE, 2002. 95 p. 
Rosinha, R. O. Estratégias competitivas e reestruturação da indústria de sementes no Brasil: a análise do segmento do milho. Dissertação (Mestrado em Mercadologia e Administração Estratégica) - Universidade Federal de Minas Gerais, Lavras. Ano de obtenção: 2000.

Sangoi, L; Ernani, P. R; Silva, P. R. F. da; Horn, D; Schmitt, A; Schweitzer, C, et al. Rendimento de grãos e margem bruta de cultivares de milho com variabilidade genética contrastante em diferentes sistemas de manejo. Ciência Rural, v. 36, n. 3, p. 747-755, 2006.

Sangoi, L; Silva, P. R. S; Schweitzer, C; Schimitt, A; Gracietti, M. A; Motter, F, et al. A rentabilidade de sistemas de produção contrastantes quanto ao investimento em manejo dependente da base genética da cultivar de milho utilizada. In: REUNIÃO TÉCNICA ANUAL DO MILHO, 49., REUNIÃO TÉCNICA ANUAL DO SORGO, 32., 2004, Porto Alegre. Anais. Porto Alegre: FEPAGRO: EMATERRS. 1 CD-ROM.

Santos, P. G; Juliatti, F. C; Buiatti, A. L; Hamawaki, O. T. Avaliação do desempenho agronômico de híbridos de milho em Uberlândia, MG. Pesquisa Agropecuária Brasileira, v. 5, n. 37, p. 597-602, 2002.

Silva, A. A; Silva, P. R. F; Argenta, G; Sangoi, L; Minetto, T.; RAMBO, L.; SUHRE, E.; STRIEDER, M. Desempenho agronômico e econômico de tipos de cultivares de milho em função de níveis de manejo. In: CONGRESSO NACIONAL DE MILHO E SORGO, 25., 2004, Cuiabá. Resumos... Sete Lagoas: ABMS/Embrapa Milho e Sorgo/Empaer, 2004. p. 303.

Zanatta, J. C; Schiocchet, M. A; Nadal, R. Mandioca consorciada com milho, feijão ou arroz de sequeira no Oeste Catarinense. Florianópolis: Empresa de Pesquisa Agropecuária e Difusão de Tecnologia de Santa Catarina. 1993. 36 p. (Boletim Técnico).

Recebido: 08/07/2013

Received: 07/08/2013

Aprovado: 28/10/2013

Approved: 10/28/2013 